\title{
Hablemos hoy de tabaquismo
}

Juan W Zinser, Dr. ${ }^{(1)}$

\begin{abstract}
$\mathrm{A}^{\mathrm{p}}$ pesar de las graves consecuencias derivadas del abaquismo, que lo han convertido en la causa evitable de enfermedad y muerte más importante en muchos países, todavía más de $20 \%$ de los médicos en México fuman y el respeto a las instituciones de salud como espacios $100 \%$ libres de humo de tabaco no se logra plenamente. Es probable que, muchos médicos no tengan claro que la medida preventiva más importante en salud pública es no fumar (hay otras medidas también básicas que ya se han alcanzado, como la implementación del sistema de drenaje y la vacunación, por dar algunos ejemplos). Tampoco parecen saber que la decisión más importante en materia de salud que una persona puede tomar es si fuma o no.

En los últimos años se han dado pasos importantes en México en el control del tabaquismo. Se ha legislado en favor de espacios públicos cerrados como áreas $100 \%$ libres de humo de tabaco y de otras medidas que establece el Convenio Marco para el Control del Tabaquismo de la Organización Mundial de la Salud, como publicidad, promoción, venta, pictogramas, impuestos, etc. Los resultados, hasta el momento, son parciales; las leyes son subóptimas y su cumplimento inadecuado, con importantes diferencias en su aplicación en distintas entidades del país. No obstante, ha mejorado la conciencia de la población sobre los daños secundarios que provoca el tabaquismo y sobre la necesidad de proteger a los no fumadores. La importancia de respetar las leyes repercute positivamente en la calidad de vida no sólo de las personas que no fuman sino también de los
\end{abstract}

fumadores y, en general, se puede decir que el entorno social en relación al tabaquismo es mejor ahora que hace 2 años. La experiencia de otros países que nos han antecedido en el control efectivo del tabaquismo indica que el número de fumadores en la población general decrece lentamente, donde $1 \%$ de disminución al año es una cifra satisfactoria. ${ }^{1}$ Sin embargo, en países como Estados Unidos, la incidencia de tabaquismo entre los médicos ha descendido de 1 a $2 \%$, en contraste con más de $50 \%$ de médicos fumadores a mediados del siglo pasado. Las medidas para el control del tabaquismo incrementan su efectividad si el médico apoya y asesora de manera permanente a sus pacientes al respecto.

En lugar de ver la adicción a la nicotina como una de las más graves que existe, prevalece en la sociedad la aceptación del tabaquismo como un "estilo de vida" y una "costumbre" que incluye a personas de ambos sexos, de todas las edades y de todos los sectores económicos y laborales. Muchos médicos, enfermeras y diferentes trabajadores de la salud también muestran esta actitud permisiva hacia el tabaquismo, aun sin ser fumadores. Un reflejo de esta actitud es el hecho de que todavía en algunos hospitales, el tabaquismo se incluye, irónicamente, en las historias clínicas como parte de los antecedentes personales no patológicos. Además de las campañas encaminadas a controlar el tabaquismo, el consejo médico cotidiano representa una gran ayuda para que los pacientes y sus familiares abandonen el consumo de tabaco. Desafortunadamente, cuando ocurre lo contrario se tiene un impacto negativo en el control de

(I) Consejo Mexicano Contra el Tabaquismo, Instituto Nacional de Cancerología. México.

Fecha de recibido: 4 de junio de 2010 - Fecha de aceptado: 9 de junio de 2010 Solicitud de sobretiros: Dr. Juan W. Zinser. San Fernando 22. 14000 Tlalpan, México, DF. Correo electronico: juanwzinser@infosel.net.mx 
la epidemia; si el médico que está consciente de que un paciente y/o sus familiares fuman no interviene con un consejo antitabáquico y se mantiene al margen, está favoreciendo que el fumador persista en su adicción pues este último percibe esta actitud como una aprobación o cierta protección de parte del primero..
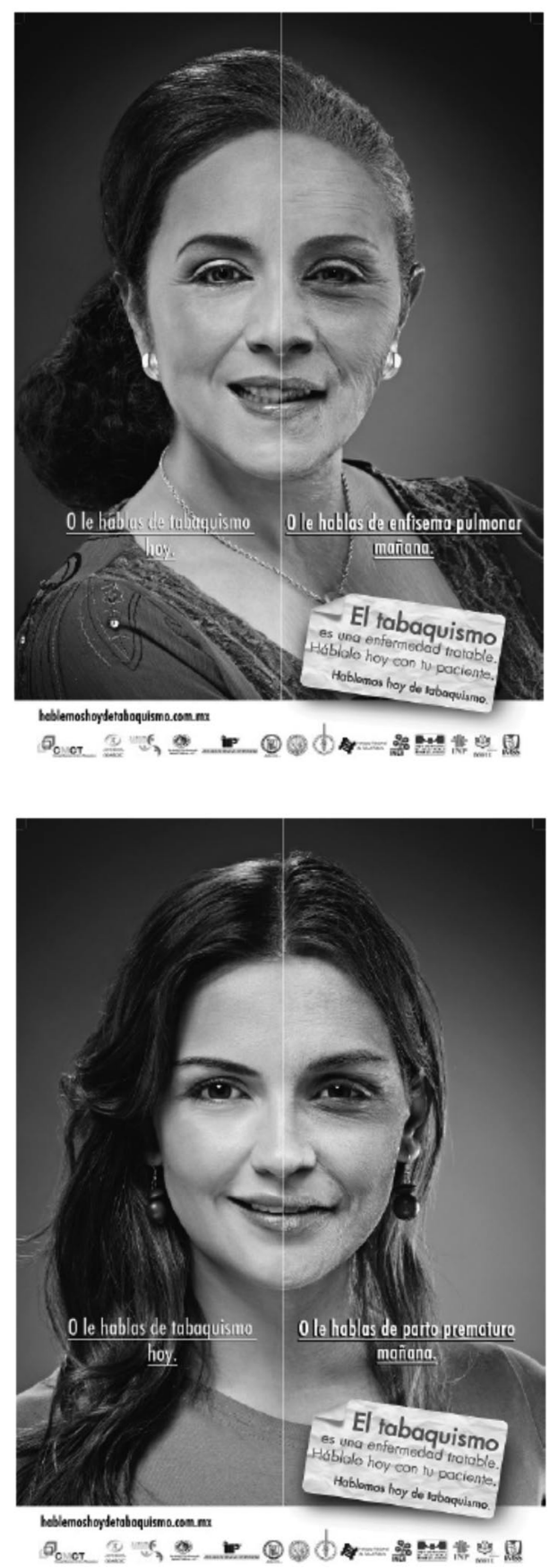

Se ha visto que si el médico proporciona a sus pacientes y acompañantes un breve consejo médico de unos cuantos minutos de duración durante la consulta, $5 \%$ de la población fumadora que recibe este consejo deja de fumar anualmente. El poder de este consejo tiene una doble función: por un lado, proporciona la información
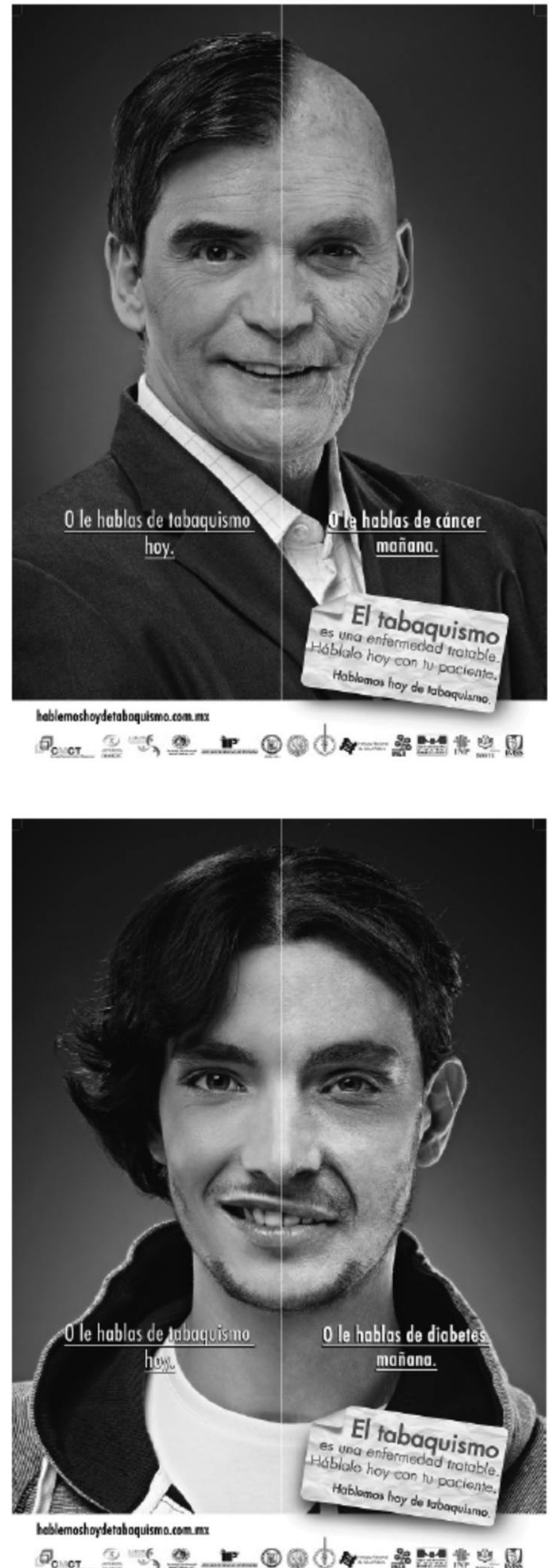

Figura I. Carteles de la campaña Hablemos hoy de tabaquismo 
relacionada con los daños derivados del tabaquismo y con los beneficios obtenidos al dejar de fumar, y por otro lado, logra que el paciente tenga la percepción de que su médico no es ajeno al tema y se interesa por su bienestar, aun cuando el motivo de la consulta no se haya relacionado con alguna patología del consumo de tabaco. Es por esto que los médicos no podemos tener una actitud indiferente en relación con el tabaquismo y sus consecuencias o ante la importancia de la cesación, ni ser neutrales en el tema ya que nuestra posición, ya sea activa o pasiva, siempre tiene un efecto.

Tanto en México como en otros países, es preocupante el alto índice de tabaquismo en los médicos, situación que obviamente influye en la limitada capacidad de los mismos de transmitir la información necesaria, $\mathrm{o}$ algún consejo afortunado como mínimo, a los pacientes para que logren dejar de fumar. El 70\% de los pacientes fumadores que acuden a consulta médica no están recibiendo la ayuda que necesitan para dejar de fumar. Las campañas generales para el control del tabaquismo y el grado de conciencia que existe al respecto no han sido suficientes para incrementar y mejorar la participación del médico en el control del tabaquismo. La necesidad de modificar la actitud médica en el control del tabaquismo condujo al Consejo Mexicano Contra el Tabaquismo (CMCT) a crear la campaña denominada Hablemos hoy de tabaquismo. Dicha campaña está dirigida a incentivar a los diferentes profesionales de la salud a que desempeñen un papel activo de ayuda a sus pacientes fumadores para que dejen de fumar. Con esto se busca proporcionarles información sobre las consecuencias de no abordar el tema del tabaquismo en estos momentos tan importantes de la prevención y concientizarlos acerca de los enormes beneficios que se pueden alcanzar hoy al prevenir el EPOC, el infarto, los aneurismas, el cáncer ...

La campaña Hablemos hoy de tabaquismo está constituida por dos instrumentos de comunicación:

1. El uso de carteles como los que se muestran en la figura 1 que ilustran el cambio que se puede producir en una persona si no deja de fumar. Estos carteles se han distribuido en diferentes instituciones de salud con la intención de que se coloquen en consultorios y áreas visibles para público.

2. La página www.hablemoshoydetabaqusmo.com. mx que incluye dichos carteles y que proporcionará además información amplia sobre el tabaquismo y las patologías derivada del mismo, así como las diferentes alternativas que existen para tratarlo. Con esta página también se busca facilitar la navegación del lector en el tema a través de diferentes vínculos relacionados. Este espacio buscará ser además un vehículo dinámico de comunicación recíproca con los lectores, ya que se pretende incorporar continuamente comentarios e información que faciliten y enriquezcan el diálogo entre los profesionales de la salud y los pacientes.

La campaña Hablemos hoy de tabaquismo es una iniciativa del Consejo Mexicano Contra el Tabaquismo que ha reunido la valiosa participación de las siguientes instituciones:

Consejo Nacional contra las Adicciones, Instituto Mexicano del Seguro Social, Instituto de Seguridad y Servicios Sociales de los Trabajadores del Estado, Instituto Nacional de Enfermedades Respiratorias Ismael Cosío Villegas, Instituto Nacional de Salud Pública, Instituto Nacional de Pediatría, Instituto Nacional de Psiquiatría Ramón de la Fuente, Instituto Nacional de Cancerología, Instituto Nacional de Ciencias Médicas y Nutrición Salvador Zubirán, Alianza Nacional para el Control del Tabaco, Asociación Mexicana de Pediatría, A.C., Sociedad Mexicana de Neumología y Cirugía de Tórax, A.C. y Sociedad Mexicana de Salud Pública, A.C. Se espera que además se incorporen otras instituciones federales y estatales para que en el curso de los próximos meses participen la gran mayoría de las instituciones de salud del país.

El tabaquismo tiene graves consecuencias médicas y económicas; pero también es consecuencia derivada del mismo la necesidad de tratarlo y prevenirlo. No es suficiente con señalar el daño que causa sino que hay que trabajar activamente y apoyar mejor y de manera integral a los pacientes en su tratamiento y prevención.

\section{Declaración de conflicto de intereses}

Declaro no tener conflicto de intereses.

\section{Referencias}

I. Organización Mundial de la Salud. Reporte Mundial de la OMS sobre la Epidemia del Tabaquismo, 2008-paquete MANPOWER. Ginebra: OMS, 2008. 